\title{
Satisfacción laboral del personal de servicios de alimentos y bebidas en hoteles de La Habana
}

\section{Job satisfaction of food and beverage service personnel in hotels in Havana}

Beatriz romaní Bendig. ${ }^{1}$, Julia Espinosa Manfugas. ${ }^{2}$ René Tejedor Arias ${ }^{3}$, Luisa Pérez Cabelleira ${ }^{4} \&$ Sandra García Vitier ${ }^{5}$

\begin{abstract}
The present study was carried out in two Havana hotels belonging to one of the hotel groups of the Ministry of Tourism, hereinafter hotel A and hotel B, with the objective of determining the satisfaction and motivation of the staff working in the areas of Food and beverage services. The work procedure developed included the application of a questionnaire to personnel working in these areas. The data was processed based on the calculation of percentages, the two-dimensional Friedman test and the Wilcoxon test to determine if there is a significant difference between work priorities. The optimal scaling technique and Cronbach's alpha were also used for the evaluation of the reliability of the instrument, through the statistical package Statistic Program for Social Sciences "(SPSS) for Windows (version 22.0). It was determined that workers feel committed to their work. The recognition of work, self-improvement, promoting teamwork, and the work group are the variables that most affect staff satisfaction.
\end{abstract}

Key Words: Job Satisfaction, Food, Beverages, Optimal Scaling

\section{Resumen}

El presente estudio se realizó en dos hoteles de La Habana pertenecientes a uno de los grupos hoteleros del Ministerio de Turismo, en lo adelante hotel A y hotel B, con el objetivo de determinar la satisfacción y motivación laboral del personal que labora en las áreas de servicios de alimentos y bebidas. El procedimiento de trabajo desarrollado

\footnotetext{
${ }^{1}$ Máster en Ciencia y Tecnología de los Alimentos. Profesora Auxiliar. Facultad de Turismo. Universidad de La Habana, Cuba. bea_romani@ftur.uh.cu

${ }^{2}$ Doctora en Ciencias Alimentarias (PhD). Profesora Titular. Facultad de Turismo. Universidad de La Habana, Cuba, julia_espinosa@ftur.uh.cu.

${ }^{3}$ Doctor en Ciencias Alimentarias (PhD). Profesor Titular. Instituto Farmacia Alimentos. Universidad de La Habana, Cuba. tejedor@ifal.uh.cu.

${ }^{4}$ Licenciada en Economía, Universidad de La Habana. Directora de Calidad del Ministerio de Turismo, Habana, Cuba. E-mail: luisa.perez@mintur.gob.cu

${ }^{5}$ Licenciada en Turismo. Universidad de La Habana, Cuba.
} 
incluyó la aplicación de un cuestionario al personal que labora en estas áreas. El procesamiento de los datos se realizó a partir del cálculo de porcentajes, el test bidimensional de Friedman y prueba de Wilcoxon para determinar si existe diferencia significativa entre las prioridades laborales. Se utilizó además la técnica de escalamiento óptimo y el alpha de Cronbach para la evaluación de la confiabilidad del instrumento, a través del programa estadístico Statistic Program for Social Sciences" (SPSS) para Windows (versión 22.0). Se determinó que los trabajadores se sienten comprometidos con su labor. El reconocimiento del trabajo, la superación personal, promover el trabajo en equipo, y el colectivo de trabajo son las variables que más inciden en la satisfacción del personal.

Palabras Clave: Satisfacción Laboral, Alimentos, Bebidas, Escalamiento Óptimo.

\section{Introducción}

Según la Organización Mundial del Turismo (OMT), en el 2018 se alcanzó la cifra de 1,400 millones de arribos de turistas internacionales, representando un aumento del $6 \%$ con respecto al año anterior. De esa manera el año 2018, consolidó los fuertes resultados alcanzados en el 2017, significando así el segundo mejor año desde el 2010 y constituyendo el noveno año consecutivo de aumento en el arribo de turistas internacionales, con un promedio de crecimiento del $4 \%$ por año; secuencia de crecimiento ininterrumpido no presenciado desde la década de 1960 (OMT, 2017; OMT, 2019).

La actividad de restauración constituye un eslabón de gran importancia dentro de la industria turística y es reconocida como uno de los elementos que mayores ingresos reporta dentro de la industria hotelera.

Por otra parte, uno de los factores esenciales para el desarrollo sostenido de toda organización u actividad es el factor humano. La conducta del personal es determinante en la calidad del servicio a largo plazo, si bien los aspectos técnicos son de gran de importancia, los hechos demuestran que los clientes de un restaurante valoran altamente que el personal inspire confianza sin que esto quiera decir que no dominen la técnica de la profesión. En tal sentido se puede afirmar que la motivación y satisfacción de los trabajadores marcarán la experiencia del cliente.

Pocos conceptos han suscitado más interés y despertado tantas expectativas como los vinculados con los procesos motivacionales (Barbera, 1999). Estos intervienen en cualquier tipo de actividad en que participan los seres humanos; explican las causas por las cuales las personas hacen o no hacen determinadas cosas y, de hecho, al servir de fuentes inductoras y dinamizadoras de la actuación, nos acercan a la comprensión del comportamiento humano.

Robbins (1999) define la motivación como la voluntad de ejercer altos niveles de esfuerzo para alcanzar las metas organizacionales, voluntad que está condicionada por la capacidad que tiene ese esfuerzo para satisfacer alguna necesidad individual. La motivación es, en 
síntesis, lo que hace que un individuo actúe y se comporte de una determinada manera (Solanas, 1993). La motivación es un factor preponderante para la productividad y la satisfacción laboral (Ardouin, Bustos, Gayó y Jarpa, 2000).

En la comunidad científica, existe consenso en señalar que la satisfacción laboral es la actitud que asume la persona ante su trabajo y que se refleja en actitudes, sentimientos, estados de ánimo y comportamientos en relación a su actividad laboral. Si la persona está satisfecha con su trabajo responderá adecuadamente a las exigencias de este; si, por el contrario, está insatisfecha no será capaz de realizar su labor con eficiencia y calidad. Además, la insatisfacción laboral se refleja en todas las esferas de la vida del trabajador (García, 2010).

No obstante, una de las definiciones más difundida de satisfacción es la propuesta por Davis y Newstrom (2003), que plantea que la satisfacción laboral es un concepto multidimensional que agrupa diversos factores, destacando aquellos que están directamente relacionados con la naturaleza de la tarea desempeñada y aquellos relativos al contexto laboral (el ambiente de trabajo, los compañeros, etc.).

En tal sentido, cualquier empresa que se precie y que tenga como objetivo aumentar sus rendimientos deberá tener presente tanto la motivación como los aspectos satisfactorios de sus trabajadores. No hay que olvidar que el mayor activo de una empresa es su capital humano (Zubiri, 2013).

Lo anterior, unido al compromiso de la alta dirección del Ministerio de Turismo (Mintur) y de los grupos hoteleros de elevar la competitividad del destino Cuba y en particular los servicios de alimentos y bebidas por su implicación en la actividad turística, ha promovido la realización de este estudio, en dos hoteles de La Habana, hotel A con categoría 5 estrellas y hotel B con categoría 4 estrellas, ambos pertenecen al mismo grupo hotelero.

De ahí que la investigación tuvo como objetivo determinar la satisfacción y motivación laboral del personal que labora en las áreas de servicios de alimentos y bebidas de los hoteles objeto de estudio.

\section{Metodología empleada}

Para conocer el nivel de motivación y satisfacción laboral del cliente interno de los hoteles objeto de estudio se aplicó un cuestionario adaptado a partir del propuesto por Monet (1995) y el reportado por González (2015) al personal de cocina y servicio gastronómico de ambos establecimientos. El instrumento aplicado estuvo conformado por 20 indicadores relacionados con las condiciones laborales de los trabajadores, la motivación y relación profesional con los directivos inmediatos, así como la satisfacción con la labor que realizan, el colectivo laboral y el estilo de trabajo de los directivos. Contempla además datos demográficos de los encuestados y otros relativos a su formación y capacitación. 
El tipo de diseño utilizado en la investigación fue transversal descriptivo, ya que se recolectaron los datos en un tiempo único con el fin de describir el fenómeno y analizar su incidencia en un momento dado (Hernández, Fernández y Baptista, 2010). Se realizó un muestreo probabilístico con un 95\% de nivel de confianza, para esto se utilizó el Sample Size Calculator (2007). La Tabla 1 muestra un resumen sobre los datos de la aplicación de la encuesta.

Tabla 1. Ficha técnica sobre la aplicación de la encuesta al cliente interno

\begin{tabular}{rcr}
\hline & Hotel A & Hotel B \\
\hline Universo & 150 & 71 \\
Tamaño muestral & 66 & 52 \\
Nivel de confianza & $95 \%$ & $95 \%$ \\
Margen de error & $9 \%$ & $7 \%$ \\
Forma de contacto & Personal \\
Punto de muestreo & Área de alimentos y bebidas \\
\hline
\end{tabular}

Para estimar la confiabilidad de la encuesta aplicada al cliente interno se determinó el coeficiente Alfa de Cronbach. Se trata de un índice de consistencia interna que toma valores entre 0 y 1 . Para su interpretación se consideró lo reportado por Hernández, Fernández y Baptista (2010), que establece que si el valor del coeficiente es de 0.25, indica baja confiabilidad; si el resultado es 0.50 , la fiabilidad es media o regular; si supera el 0.75 es aceptable, y si es mayor a 0.90 es elevada.

El análisis de los resultados se realizó mediante la estadística descriptiva a partir del cálculo del porcentaje. En el caso de la pregunta relacionada con las prioridades por las que trabajan, se procesó a partir del test de Friedman y el estadístico Wilcoxon.

- Test bidimensional de Friedman y prueba de Wilcoxon

Con el objetivo de determinar si existe diferencia significativa entre las prioridades laborales para los trabajadores, se empleó la prueba no paramétrica de ordenamiento por rangos, procesando los resultados mediante el análisis bidimensional de Friedman considerando un nivel de confianza del 95\% $(\alpha=0.05)$, posteriormente, se procedió a realizar la prueba de Wilcoxon para determinar entre cuáles de las variables analizadas existía diferencia significativa para un nivel de confianza del $95 \%$.

Se utilizó además la técnica de escalamiento óptimo con el fin de obtener una representación gráfica que permita visualizar las variables que más caracterizan el fenómeno estudiado, ya que esta técnica trata de representar en un espacio geométrico de escasas dimensiones (dos o tres) las proximidades existentes entre un conjunto de objetos y sujetos, a la vez que reduce la dimensionalidad de los datos, minimizando la función de pérdida de información (Correa, 2008). 


\section{Resultados}

\section{Caracterización de la muestra}

La Tabla 2 muestra una caracterización del personal que labora en las áreas de alimentos y bebidas de los hoteles objeto de estudio. Como se aprecia, existe un predominio del sexo masculino (68\%). El rango de edad más representado en la muestra es el de 41 a 60 años con un 61,6\%, mientras que entre 21 a 40 años se ubica el 34,4 \%. Los trabajadores mayores de 60 años representan el 2,4 \% y los menores de 20 años solo el 0,8 \%, lo que evidencia que dentro de la fuerza laboral de estos hoteles no predominan los jóvenes.

Al analizar el nivel de escolaridad se observa que la mayoría representada por $44,8 \%$ tiene el nivel medio superior vencido, y el 34,4\% refieren haber cursado un técnico medio. Por su parte, con nivel superior labora un 16,8 \% de los encuestados. La mayor parte de los encuestados (79 \%) afirman tener formación especializada en hotelería y turismo. Es de gran beneficio que la fuerza laboral esté capacitada para realizar su trabajo ya que dispone de habilidades que permiten un mejor desempeño y, por ende, una mejor calidad de los servicios. Al observar los años de experiencia laboral se aprecia un predominio en el rango de 16 a 20 años con un $32 \%$, seguido de un equilibrio entre los rangos de 11 a 15 años y más de 20 años, lo que lleva a pensar que, de manera general, son personas con experiencia y conocedoras de la labor que realizan.

El 92,8 \% de los trabajadores expresa sentirse capacitado para realizar la labor que realiza, sin embargo, los encuestados declaran la necesidad de recibir cursos de actualización para conocer sobre las nuevas tendencias de la restauración ya que según plantean se desempeñan en un área que evoluciona constantemente y donde los clientes son cada vez más exigentes y conocedores, por lo que satisfacerlos requiere de una mejor preparación, que incluya brindar un servicio con la calidad del servicio requerida.

Tabla 2. Caracterización de la muestra de trabajadores encuestados.

\begin{tabular}{clr}
\hline & & \\
Variables & Categorías de respuesta & $\%$ \\
\hline Sexo & Femenino & 32 \\
& Masculino & 68 \\
\hline Área & Cocina & 43.2 \\
& Servicios & 56.8 \\
\hline Edad & Menos de 20 & 0.8 \\
& 21 a 40 & 34.4 \\
& 41 a 60 & 61.6 \\
& Más de 60 & 2.4 \\
\hline Nivel escolar & 9no Grado & 0.8 \\
& Técnico Medio & 34.4 \\
& 12mo Grado & 44.8 \\
& Superior & 16.8 \\
\hline Formación & Sí & 79.2 \\
especializada & No & 14.4 \\
\hline Años de experiencia & Menos de 1 año & 3.2 \\
& 1 a 5 & 17.6
\end{tabular}




\begin{tabular}{llr} 
& 11 a 15 & 24 \\
& 16 a 20 & 32 \\
& Más de 20 & 21.6 \\
\hline Capacidad para & Sí & 92.8 \\
realizar la labor & No & 0.8 \\
& No sé & 1.6 \\
\hline
\end{tabular}

\section{Comportamiento de indicadores de las dimensiones evaluadas}

La Figura 1 muestra los resultados de las opiniones de los encuestados en cuanto a las condiciones laborales. Como se puede observar, los resultados del hotel A son superiores para todos los indicadores. La variable más deteriorada tanto a nivel general (47\%) como en cada uno de los hoteles es la transportación; seguida de equipamientos e insumos (62 $\%)$ y de condiciones de trabajo (65\%). Con respecto al transporte, esto pudiera estar condicionado porque, aunque se dispone de transporte obrero, sus horarios no se ajustan del todo a los horarios de los trabajadores de servicio. Los equipamientos e insumos están más afectados en el hotel B; se pudo conocer que existen problemas con las cámaras frías, la campana de extracción e insuficiente cantidad de vajillas.

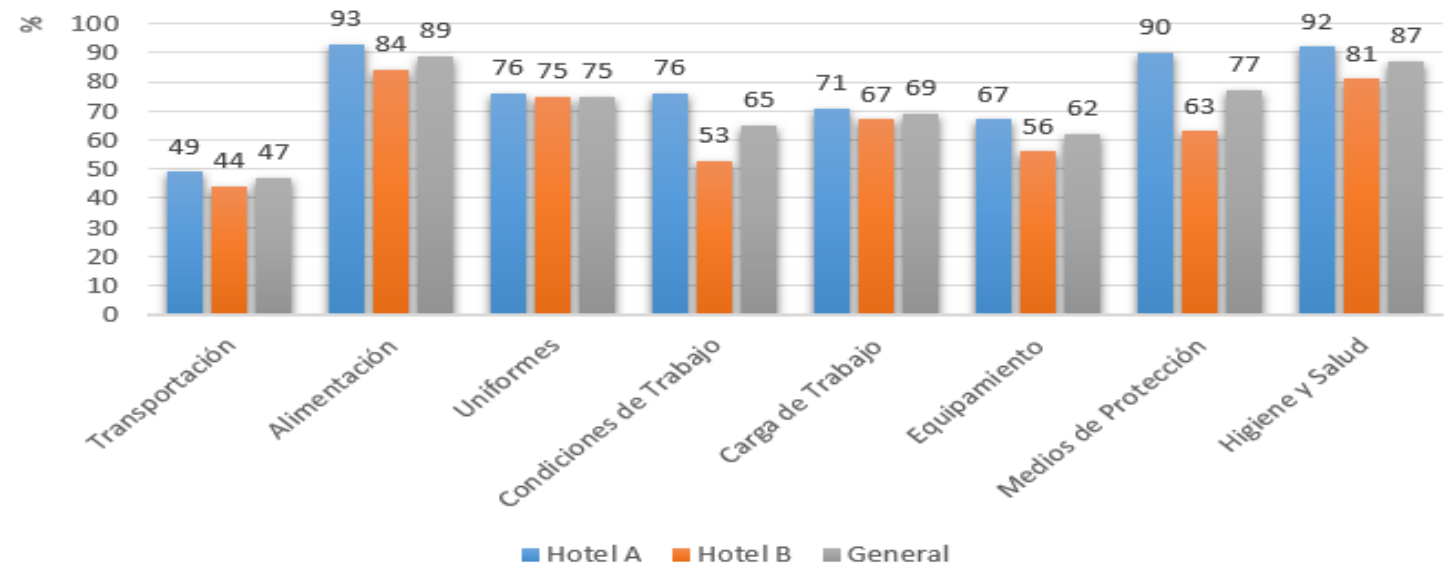

Figura 1. Resultados de las condiciones laborales de los trabajadores

La variable uniforme reporta un $75 \%$ de cumplimiento, dado por el tiempo que transcurre entre una entrega y otra a los trabajadores, lo que evidentemente afecta su satisfacción, aunque debe señalarse que, durante la realización del presente estudio, fueron entregados al personal de cocina uniformes en el hotel A. Con mejores resultados aparecen la alimentación (89\%) y la higiene y salud en el trabajo (87\%). Asimismo, los medios de protección mostraron resultados positivos en el caso del hotel A, mientras que en el B los trabajadores manifestaron necesidad de guantes de malla metálica para las operaciones de corte en la carnicería y calzado de seguridad con suela antideslizante. En cuanto a la carga de trabajo los valores de satisfacción son bajos, algunos empleados plantearon que en ocasiones se hace necesario extender la jornada por diferentes motivos.

Los resultados obtenidos en la dimensión motivación y relación profesional con los directivos, se exponen en la Figura 2. Como se aprecia, de manera general los trabajadores 
expresan insatisfacción con respecto a los estímulos morales, variable que solo alcanza un $50 \%$ de satisfacción. Además, manifiestan inconformidad en cuanto al indicador "Se escuchan y valoran sus criterios e iniciativas", que alcanzó sólo un $64 \%$ de satisfacción y "Se reconoce su trabajo" con un $67 \%$. De esta manera se evidencia que los trabajadores necesitan más participación en las cuestiones relativas a su área. En este sentido se debe señalar que el estímulo y reconocimiento moral, es fuente de incentivo, el reconocimiento de buenas prácticas estimula el esfuerzo realizado y alienta a la obtención de nuevos objetivos, metas más altas, en fin, al desarrollo profesional del trabajador y su empresa.

Con respecto al reconocimiento de los directivos a sus trabajadores, Clavería (2009) reporta que si la empresa no empieza por cumplir con quienes le permiten competir, mejorar, innovar y cambiar, difícilmente podrá esperar algún cambio. Premiar la labor de los empleados, es un acto de reconocimiento no tiene que ser tan costoso para ser inolvidable.

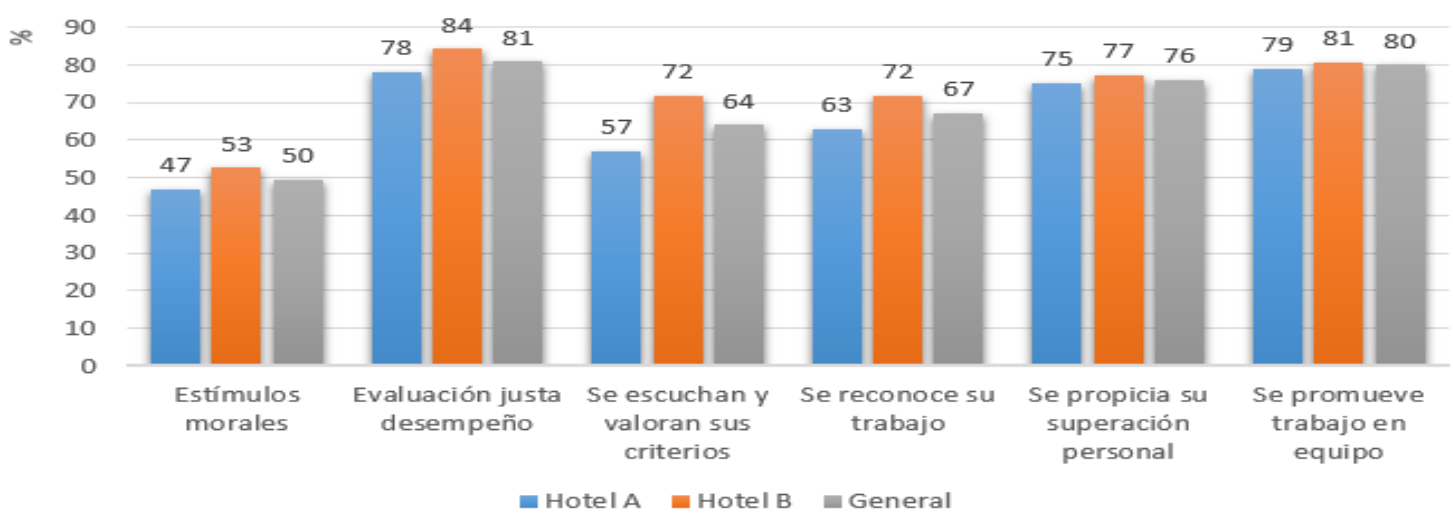

Figura 2. Resultados de la motivación y relación profesional con los directivos inmediatos

Los aspectos con los cuales el personal demuestra sentirse más complacido son la evaluación del desempeño de forma justa y el trabajo en equipo, alcanzando valores de $81 \%$ y $80 \%$ respectivamente. Para Chiavenato (2007) las relaciones interpersonales incrementan la confiabilidad de liderazgo y la unión de sus miembros, aumentando la motivación y reforzamiento entre el personal, logrando un comportamiento responsable y de compromiso; en sintonía con la productividad de la organización. Es importante una comunicación efectiva en la que sea relevante la compresión, el respeto y la ayuda mutua entre los trabajadores para superar debilidades, afianzar fortalezas que redunden en la convivencia, el crecimiento de las personas la calidad de su desempeño y el beneficio de quienes reciben sus servicios (Werther, 2000).

De las dos instalaciones estudiadas el hotel A mostró los valores más bajos de motivación en todos estos aspectos, el cual es operado como empresa mixta. Sería interesante valorar, entonces, si la naturaleza del contrato de administración pudiera estar incidiendo en estos resultados. Al analizar lo anterior es fundamental recordar la necesidad de disponer de métodos más participativos en los cuales los trabajadores tengan un mayor protagonismo, promover la comunicación entre los diferentes actores de las entidades y tomar en 
consideración los criterios del personal como parte fundamental en el logro de los objetivos de la empresa.

La Figura 3 ilustra los valores de la satisfacción laboral con un grupo de indicadores, el porcentaje de satisfacción general con la labor que realizan es de $82 \%$, se aprecia que el $80 \%$ de los encuestados considera sentirse satisfecho con el colectivo de trabajo y el $75 \%$ con el centro de trabajo. Por otra parte, el estilo de trabajo de los directivos es bajo, alcanzó solo un $47 \%$ de satisfacción de manera general, resultando una variable deteriorada, en opinión de algunos encuestados debe existir mayor comunicación entre los directivos y los trabajadores con el objetivo de que se tengan en cuenta las opiniones y criterios, aspecto que no obtuvo un buen resultado en la dimensión "Motivación y relación con los directivos inmediatos".

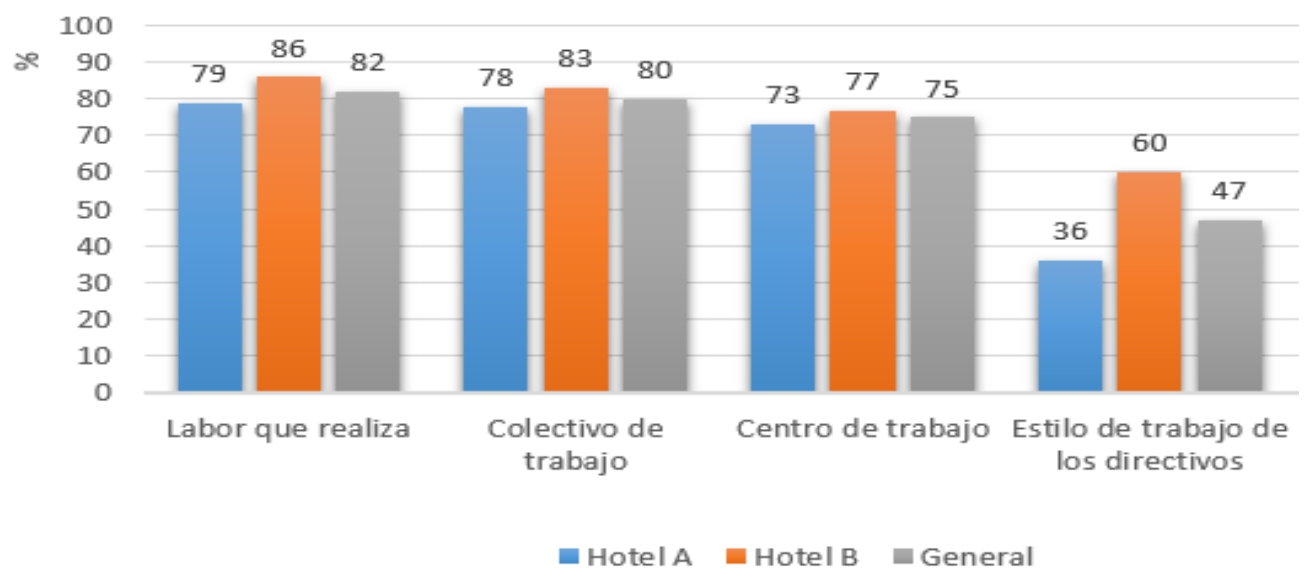

Figura 3. Resultados de la satisfacción laboral

Se debe reflexionar al respecto, pues no es suficiente contar con trabajadores comprometidos, satisfechos con la labor que realizan y a su vez con su centro y colectivo de trabajo, si no existe un liderazgo compartido con estilos de dirección altamente participativos.

Los resultados acerca del nivel de compromiso de los trabajadores, se ilustran en la Figura 4. La mayor parte de los encuestados afirma sentirse muy comprometido o comprometido (89\%) con relación a su labor dentro del hotel, esto evidencia el alto sentido de pertenencia de estos trabajadores, los cuales a pesar de mostrar determinadas insatisfacciones revelan su compromiso con lo actividad que desempeñan.

Este es un resultado muy favorable, según Mehech et al. (2016) las empresas y organizaciones con un alto nivel de compromiso presentan niveles de productividad de sus trabajadores hasta $78 \%$ sobre las que tienen un bajo nivel de compromiso de su fuerza laboral. 


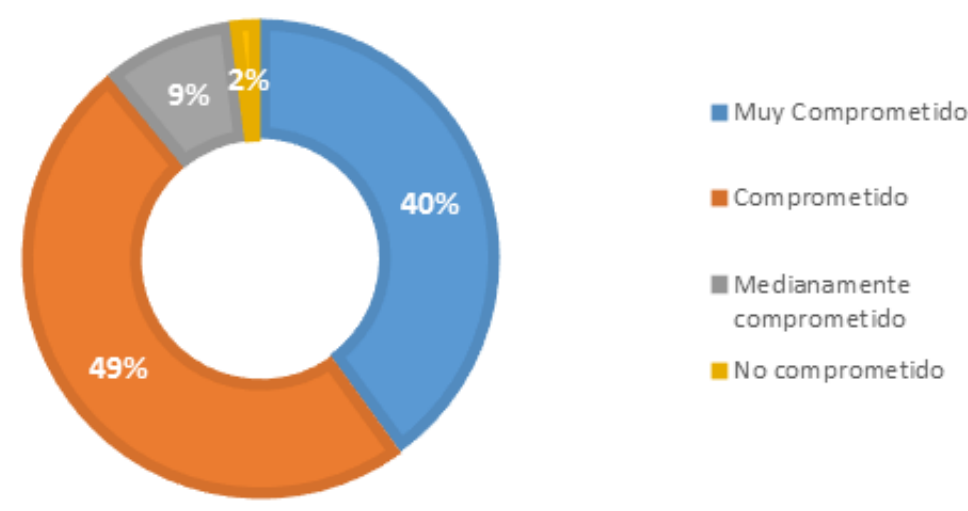

Figura 4. Resultados acerca del nivel de compromiso de los trabajadores

La Tabla 3 muestra las prioridades de los trabajadores luego de aplicar el test de Fridman y prueba de Wilcoxon. Como se puede apreciar el salario constituye lo más importante, pero no se establece una diferencia significativa $(\mathrm{p}<0.05)$ con respecto a las variables necesidad económica, lo útil y necesario del trabajo, la seguridad del empleo y el ambiente y las relaciones de trabajo, pues las cinco se encuentran en un primer orden de prioridad. Este resultado coincide con los obtenidos por Lorenzo (2015).

Tabla 3. Resultados de las prioridades de los trabajadores

\begin{tabular}{ll}
\hline \multicolumn{1}{c}{ Variables } \\
\hline Salario recibido & $\mathrm{a}$ \\
Necesidad económica & $\mathrm{a}$ \\
Útil y necesario del trabajo & $\mathrm{a}$ \\
Seguridad de empleo & $\mathrm{a}$ \\
Ambiente y relaciones de trabajo & $\mathrm{a}$ \\
Posibilidad de ser promovido & $\mathrm{b}$ \\
Reconocimiento social & $\mathrm{b}$ \\
\hline les indican que no existe diferencia significativa para $\mathrm{p}<0.05$.
\end{tabular}

Fuente: SPSS, versión 22.0

Por su parte, no existe diferencia significativa entre las variables posibilidad de ser promovido y reconocimiento social, situándose en un segundo nivel de prioridad. Llama la atención que el reconocimiento social sea de menor prioridad, al decir de Loor (2015) el ser reconocido por la sociedad es muy importante, pues impulsa a los individuos a ser mejores cada día, los motiva a seguir adelante y a dar lo mejor en cada una de sus actividades, cuando son reconocidos se sienten apreciados por los demás y poseen una actitud positiva, mayor confianza en ellos mismos, ya que son muy conscientes de las habilidades que tienen y saben sacarle provecho. Por su parte Lumesse (2017) apunta que cuando el reconocimiento social forma parte de la organización, el ambiente de trabajo mejora, con todo lo que ello conlleva de aumento de la productividad y la eficiencia.

En tanto García (2012), plantea que los factores que inciden en la motivación laboral son: la retribución económica, la responsabilidad en el trabajo, el reconocimiento dentro de la empresa y el reconocimiento social. 


\section{Resultados de la técnica de escalamiento óptimo}

A continuación (Tabla 4) se presenta el análisis reduciendo el espacio de variables total a sólo dos dimensiones de la solución, valor predeterminado, ya que esta es la más sencilla para interpretar los resultados (Díaz y Garrido, 2015).

Tabla 4. Resumen del modelo

\begin{tabular}{lrr}
\hline Dimensión & $\begin{array}{c}\text { Alfa de } \\
\text { Cronbach }\end{array}$ & \multicolumn{1}{c}{ Inercia } \\
\hline 1 &, 918 &, 380 \\
2 &, 887 &, 308 \\
Total & &, 695 \\
\hline
\end{tabular}

Fuente: SPSS, versión 22.0

Las dos dimensiones seleccionadas presentan un buen nivel de fiabilidad ( $\alpha$ de Cronbach, de 0,918 y 0,887 respectivamente), en el primer caso se considera elevada y en el segundo aceptable a partir de lo establecido por Hernández, Fernández y Baptista (2010). Se debe subrayar que la interpretación en torno a las dimensiones establecidas depende de la capacidad explicativa (inercia) de cada una. En este caso ambas tienen una capacidad explicativa similar para interpretar la cercanía de las variables con cada eje que las representa.

Se observa que hay un $70 \%$ de la variabilidad de los datos explicada por ambas dimensiones incluidas en el modelo, $38 \%$ explicada por la primera y $31 \%$ explicada por la segunda.

Al analizar las medidas discriminantes por variable (Tabla 5) se observa cuánto discrimina cada una de ellas en la dimensión correspondiente, o sea la importancia de cada variable dentro de cada una de las dimensiones. La dimensión 1 se encuentra explicada especialmente por "colectivo de trabajo", "centro de trabajo" y "estilo de trabajo de los directivos", y la dimensión 2 por "reconocimiento del trabajo", "se propicia la superación personal" y "se promueve el trabajo en equipo".

Tabla 5. Medidas discriminantes por variable

\section{Medidas discriminantes}

\begin{tabular}{lrr}
\hline \multicolumn{1}{c}{ Medidas discriminantes } & \\
Variables & Dimensión & \\
\hline Transportación & 1 & 2 \\
Alimentación &, 295 &, 105 \\
Uniformes &, 264 &, 088 \\
Condiciones de trabajo &, 334 &, 206 \\
Carga de trabajo &, 319 &, 179 \\
Equipamiento e insumos &, 360 &, 321 \\
Medios de protección &, 311 &, 237 \\
Higiene y salud en el trabajo &, 236 &, 263 \\
Sistema de estímulos &, 386 &, 409 \\
\hline
\end{tabular}




\section{EDDigital}

ISSN: 2661-6831

www.exploradordigital.org

Enero - Marzo 2021

Vol. 5, N¹, pág. $229-243$

Evaluación del desempeño de forma justa

Escuchan y valoran criterios e iniciativas

,373

, 402

Se reconoce su trabajo

,393

, 454

Se propicia su superación personal

,283

, 554

Se promueve el trabajo en equipo

,341

, 534

Labor que realiza

,398

, 515

Colectivo de trabajo

Centro de trabajo

, 471

,221

, 581

Estilo de trabajo de los directivos

, 518

Nivel de compromiso

\section{Fuente: SPSS, versión 22.0}

Por su parte la figura 5 es una representación gráfica de las medidas discriminantes, donde, mientras mayor distancia del origen alcance la variable resulta más explicativa del fenómeno analizado, y la cercanía entre una y otra expresan su estrecha relación y a su vez la relación con la dimensión a la que se acerquen. Se observa que "se reconoce su trabajo", "se propicia su superación personal", "se promueve el trabajo en equipo", y "colectivo de trabajo" son las variables que más distantes se encuentran del origen y por tanto son las que más explican la satisfacción del cliente interno.

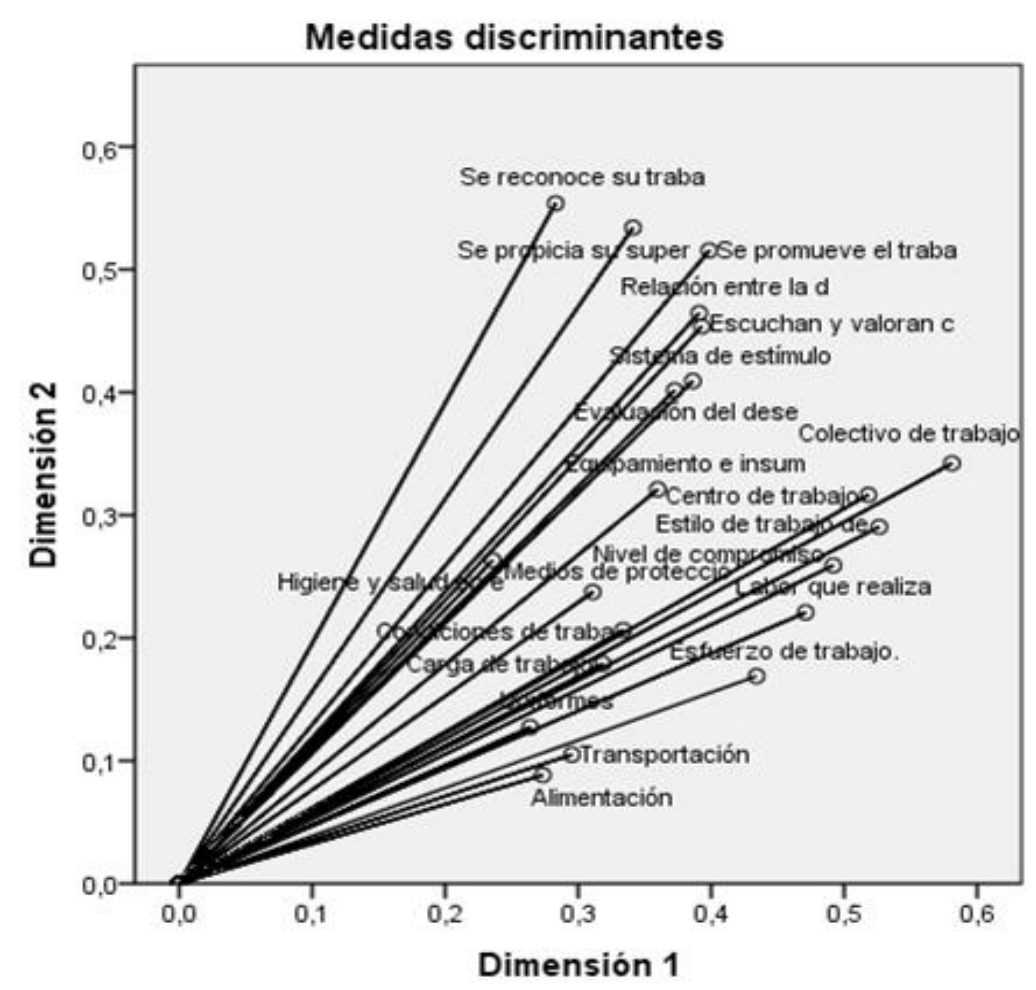

Normalización de principal de variable.

Figura 5. Representación gráfica de medidas discriminantes

Fuente: SPSS, versión 22.0

Los resultados derivados del estudio han sido presentados a la dirección de calidad del grupo hotelero correspondiente para la toma de decisiones y la propuesta de acciones de mejora. Con la implementación gradual y paulatina de estas se contribuirá al incremento 
de la motivación y satisfacción laboral y por ende al perfeccionamiento de la calidad de los servicios de alimentos y bebidas en el país.

\section{Conclusiones}

- El personal cuenta con años de experiencia en su puesto de trabajo actual, se siente comprometido y posee preparación técnica profesional; las variables con más bajo nivel de satisfacción son la trasportación, el estilo de trabajo de los directivos y los estímulos morales.

- El salario, la necesidad económica, lo útil y necesario del trabajo, la seguridad del empleo y el ambiente; y las relaciones de trabajo son las principales prioridades laborales.

- Se evidenció que el reconocimiento del trabajo, la superación personal, promover el trabajo en equipo, y el colectivo de trabajo son las variables que más explican la satisfacción del cliente interno.

- El hotel A obtuvo los mejores resultados para las variables relacionadas con las condiciones laborales de los trabajadores, mientras que las referidas a la motivación y relación profesional con los directivos inmediatos tuvieron un mejor comportamiento en el hotel B.

\section{Referencias bibliográficas}

Ardouin J., Bustos C., Gayó R. y Jarpa M (2000). Motivación y Satisfacción Laboral. Disponible en: http://www.udec.cl.

Barbera, E. (1999) Marco Conceptual e Investigación de la motivación Humana. REME. Numero1 Volumen II Disponible en: http:// reme. Uji.es.

Chiavenato, I. (2007). Administración de Recursos Humanos (Octava ed.). Mexico: Mc Graw Hill.

Correa, G. (2008). Contribuciones al análisis multivariante no lineal. (Tesis doctoral). Universidad de Salamanca, Departamento de Estadística. Recuperado de www.bdigial.unal.edu.co

Davis, K. y Newstrom, J. (2003). Comportamiento humano en el trabajo. (11ª Edición), México: McGraw Hill.

Díaz, I. y Garrido, I. (2015) Correspondencias Múltiples en SPPS. Facultad de Ciencias Sociales de la Universidad de Chile. Recuperado de https://www.ucursos.cl/facso/2015/1/SO01023/2/

Fernández, M. (1999) Diccionario de recursos humanos: organización y dirección. Madrid: Díaz de Santos. 
García, D. (2010) Satisfacción Laboral. Una aproximación teórica, en Contribuciones a las Ciencias Sociales. Recuperado de www.eumed.net/rev/cccss/09/dgv.htm

García, V. (2012). La Motivación Laboral: estudio descriptivo de algunas variables. Tesis de Grado, Universidad de Valladolid. Recuperado de: https://uvadoc.uva.es/ bitstream/10324/1144/1/TFG-B.60.pdf

González, N. Factores que inciden en la demanda del Restaurante "Salón Rosa" del Complejo Hola-Ola. Trabajo de diploma en opción al título de Licenciada en Turismo. Facultad de Turismo. Universidad de la Habana. Cuba. 2015

Hernández, R., Fernández, C., y Baptista, P. L. (2010) “Metodología de la investigación”. (3ra. ed.). Editorial McGraw-Hill Interamericana de México, S.A. de C.V. México.

Loor, A. (2015) ¿Por qué es importante el reconocimiento social? Club ensayos. Recuperado de: https://www.clubensayos.com

Lorenzo, A.I. (2015). Evaluación de la calidad de los servicios de alimentos y bebidas en el Restaurante Buffet Fausto del Hotel Plaza. Tesis en opción al Título en Licenciatura en Ciencias Alimentarias. Instituto de Farmacia y Alimentos Departamento de Alimentos. Universidad de La Habana.

Lumesse, A. 2017. La importancia del reconocimiento social en la gestión del talento. Recuperado de https://www.audalianexia.com/blog/la-importancia-delreconocimiento-social-en-la-gestion-del-talento/

Mehech, et al (2016), Medición del compromiso Laboral y su impacto en los Resultados de la Empresa, pp 1-91.

Monet, M. E. (1995) Desarrollo organizacional y consultoría. Tesis de Maestría. Instituto Farmacia-Alimentos. Universidad de la Habana. Cuba. 2015

Organización Mundial del Turismo. (2017). Informe Anual 2017. Recuperado de http://www2.unwto.org

Organización Mundial del Turismo. (2019). UNWTO World Tourism Barometer and Statistical Annex, January 2019.

Robbins, S. (1999) Comportamiento Organizacional 8va Edición Prentice Hall México

Solanas, R. (1993) Administración de Organizaciones, Ediciones interoceánicas, Buenos Aires.

Werther, J. W. (2000). Administración de Personal y Recursos Humanos. México: Mc Graw Hill. 
Zubiri, F. (2013). Satisfacción y motivación profesional. Anales del Sistema Sanitario de Navarra, 36(2), pp 193-196. Recuperado de https://dx.doi.org/ 


\section{PARA CITAR EL ARTÍCULO INDEXADO.}

Romaní Bendig, B., Tejedor Arias, R., Espinosa Manfugas, J., Pérez Cabelleira, L., \& García Vitier, S. (2021). Satisfacción laboral del personal de servicios de alimentos y bebidas en hoteles de La Habana. Explorador Digital, 5(1), 229-243. https://doi.org/10.33262/exploradordigital.v5i1.1500

\section{【 Ciencia}

El artículo que se publica es de exclusiva responsabilidad de los autores y no necesariamente reflejan el pensamiento de la Revista Explorador Digital.

El artículo queda en propiedad de la revista y, por tanto, su publicación parcial y/o total en otro medio tiene que ser autorizado por el director de la Revista Explorador Digital.
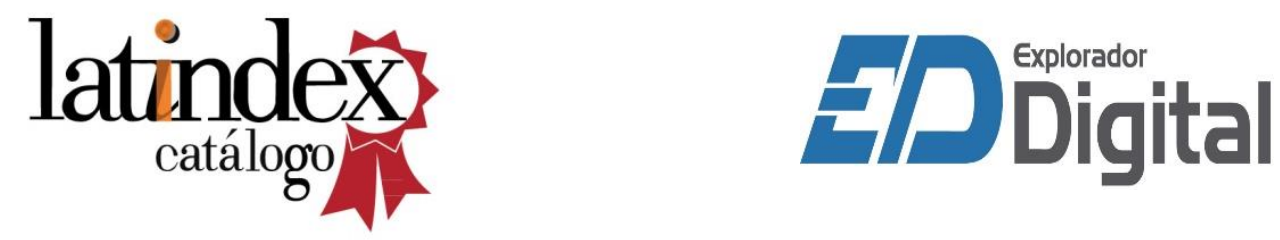